\title{
The Classification of All Crossed Products $H_{4} \# k\left[C_{n}\right]^{\star}$
}

\author{
Ana-Loredana AGORE ${ }^{\dagger \ddagger}$, Costel-Gabriel BONTEA ${ }^{\S \dagger}$ and Gigel MILITARU ${ }^{\S}$ \\ $\dagger$ Faculty of Engineering, Vrije Universiteit Brussel, Pleinlaan 2, B-1050 Brussels, Belgium \\ E-mail: ana.agore@vub.ac.be, ana.agore@gmail.com \\ URL: http://homepages.vub.ac.be/ aagore/ \\ $\ddagger$ Department of Applied Mathematics, Bucharest University of Economic Studies, \\ Piata Romana 6, RO-010374 Bucharest 1, Romania \\ $\S$ Faculty of Mathematics and Computer Science, University of Bucharest, \\ Str. Academiei 14, RO-010014 Bucharest 1, Romania \\ E-mail: costel.bontea@gmail.com,gigel.militaru@fmi.unibuc.ro,gigel.militaru@gmail.com \\ URL: http://fmi.unibuc.ro/ro/departamente/matematica/militaru_gigel/
}

Received November 18, 2013, in final form April 18, 2014; Published online April 23, 2014

http://dx.doi.org/10.3842/SIGMA.2014.049

\begin{abstract}
Using the computational approach introduced in [Agore A.L., Bontea C.G., Militaru G., J. Algebra Appl. 12 (2013), 1250227, 24 pages] we classify all coalgebra split extensions of $H_{4}$ by $k\left[C_{n}\right]$, where $C_{n}$ is the cyclic group of order $n$ and $H_{4}$ is Sweedler's 4-dimensional Hopf algebra. Equivalently, we classify all crossed products of Hopf algebras $H_{4} \# k\left[C_{n}\right]$ by explicitly computing two classifying objects: the cohomological 'group' $\mathcal{H}^{2}\left(k\left[C_{n}\right], H_{4}\right)$ and $\operatorname{CRP}\left(k\left[C_{n}\right], H_{4}\right):=$ the set of types of isomorphisms of all crossed products $H_{4} \# k\left[C_{n}\right]$. More precisely, all crossed products $H_{4} \# k\left[C_{n}\right]$ are described by generators and relations and classified: they are $4 n$-dimensional quantum groups $H_{4 n, \lambda, t}$, parameterized by the set of all pairs $(\lambda, t)$ consisting of an arbitrary unitary map $t: C_{n} \rightarrow C_{2}$ and an $n$-th root $\lambda$ of \pm 1 . As an application, the group of Hopf algebra automorphisms of $H_{4 n, \lambda, t}$ is explicitly described.
\end{abstract}

Key words: crossed product of Hopf algebras; split extension of Hopf algebras

2010 Mathematics Subject Classification: 16T10; 16T05; 16S40

Dedicated to Marc Rieffel on the occasion of his 75th birthday

\section{Introduction}

The second cohomology group $\mathrm{H}^{2}(H, A)$ classifies all extensions of an Abelian group $A$ by a group $H$, i.e. all groups $G$ that fit into an exact sequence $1 \rightarrow A \rightarrow G \rightarrow H \rightarrow 1$. More precisely, each element $f \in \mathrm{H}^{2}(H, A)$ is assigned with an extension $G_{f}$ of $A$ by $H$, namely the crossed product $G_{f}:=A \#^{f} H$ of $A$ and $H$ and the effective classification of all the extensions of $A$ by $H$ is obtain after computing the group $\mathrm{H}^{2}(H, A)$. In group theory there is a well developed cohomological machinery [1] which allows, at least for some classes of groups $A$ and $H$, to compute this cohomology group. Transferring this problem to Hopf algebras by considering group algebras over a field $k$, we obtain that any extension of $A$ by $H$ gives a coalgebra split extension of $k[A]$ by $k[H]$ in the sense of [2, Definition 1.2]. In fact, there is more: a Hopf algebra $E$ is a coalgebra split extension of $k[A]$ by $k[H]$ if and only if $E \cong k[G]$, for a group $G$ which is an extension of $A$ by $H$ [2, Example 1.4]. This can be restated in a cohomological manner as follows: $\mathcal{H}^{2}(k[H], k[A]) \cong \mathrm{H}^{2}(H, A)$, where $\mathcal{H}^{2}(k[H], k[A])$ denotes the second cohomological group for Hopf algebras introduced by Sweedler [14]. Thus, the classification of all coalgebra

\footnotetext{
${ }^{\star}$ This paper is a contribution to the Special Issue on Noncommutative Geometry and Quantum Groups in honor of Marc A. Rieffel. The full collection is available at http://www.emis.de/journals/SIGMA/Rieffel.html
} 
split extensions of a Hopf algebra $A$ by a Hopf algebra $H$ covers the extension problem from group theory. Now, if we replace the group algebra $k[A]$ by another arbitrary Hopf algebra, say a noncommutative and noncocommutative Hopf algebra such as Sweedler's 4-dimensional Hopf algebra $H_{4}$, things change radically as none of the classical cohomological techniques can be applied in this context. In other words, the cohomological type object $\mathcal{H}^{2}\left(k[H], H_{4}\right)$ needs to be computed using a direct approach.

In this paper we shall classify all coalgebra split extensions of $H_{4}$ by $k\left[C_{n}\right]$, where $C_{n}$ is the cyclic group of order $n$, i.e. all Hopf algebras $E$ that fit into a sequence $H_{4} \hookrightarrow E \stackrel{\pi}{\rightarrow} k\left[C_{n}\right]$ such that $\pi: E \rightarrow k\left[C_{n}\right]$ splits as a coalgebra map and $H_{4} \simeq E^{\operatorname{co}\left(k\left[C_{n}\right]\right)}$. Equivalently, we classify all crossed products of Hopf algebras $H_{4} \# k\left[C_{n}\right]$. This kind of crossed products are a special case of those arising in Hopf-Galois extensions theory. For a generalization of the notion of a HopfGalois extension see [9]. The fact that there is no efficient cohomology theory for arbitrary Hopf algebras led us to consider a purely computational approach which relies heavily on the methods introduced in [2]. First of all we compute the set of all crossed systems $\left(H_{4}, k\left[C_{n}\right], \triangleright, f\right)$ between $H_{4}$ and $k\left[C_{n}\right]$. This is the first computational part of our strategy, quite laborious considering the large number of axioms that need to be fulfilled by the pairs $(\triangleright, f)$ in order to provide a crossed system of Hopf algebras $\left(H_{4}, k\left[C_{n}\right], \triangleright, f\right)$. Theorem 3.1 gives the following description: the set of all crossed systems of Hopf algebras $\left(H_{4}, k\left[C_{n}\right], \triangleright, f\right)$ is parameterized by the set $\mathcal{C S}(n, k) \subseteq \mathcal{U}\left(C_{n}, C_{2}\right) \times k^{*}$, consisting of all pairs $(t, \lambda)$, where $t: C_{n} \rightarrow C_{2}$ is an arbitrary unitary map and $\lambda \in k^{*}$ is an $n$-th root of \pm 1 . Thus there are at most $n 2^{n-1}$ crossed products of the form $H_{4} \# k\left[C_{n}\right]:=H_{4 n, \lambda, t}$, for some $(t, \lambda) \in \mathcal{C S}(n, k)$ and they are described by generators and relations in Corollary 3.3 - this is the second step of our approach. Finally, the last computational step uses [2, Theorem 2.1] as a tool: we shall classify all the above crossed products by explicitly computing the classifying objects $\mathcal{H}^{2}\left(k\left[C_{n}\right], H_{4}\right)$ and $\operatorname{CRP}\left(k\left[C_{n}\right], H_{4}\right):=$ the set of types of isomorphisms of Hopf algebras of all crossed products $H_{4} \# k\left[C_{n}\right]$. The classification results are proven in Theorems 3.4 and 3.6. As an application, Corollary 3.7 provides the parametrization of $\operatorname{Aut}_{\mathrm{Hopf}}\left(H_{4 n, \lambda, t}\right)$, the group of Hopf algebra automorphisms of $H_{4 n, \lambda, t}$.

We point out that a coalgebra split extension is a special case of a more general type of Hopf algebra extension defined in [6, Definition 1.2.0]. As explained in [5, Section 5.2], it is a very difficult task to classify this general type of extensions and, to the best of our knowledge, the only example of such a classification is [10, Lemma 2.8]. There are also several known classification results for Abelian extensions of Hopf algebras associated to matched pairs of groups (see [11, 12] and the references therein). Our computational method, introduced in [2] and used in the present paper, is the most direct and natural way of approaching the classification of all coalgebra split extensions for two given Hopf algebras. This might not be the only way to approach the problem: for certain pairs of Hopf algebras $A$ and $H$, the class of all coalgebra split extensions of $A$ by $H$ can be classified by using the lifting method [8].

\section{Preliminaries}

Let $G$ and $H$ be two groups with $H$ Abelian. In what follows ord $(g)$ denotes the order of the element $g \in G,|G|$ is the order of $G$ while $\operatorname{Aut}(G)$ stands for the group of automorphisms of $G$. $\mathcal{U}(G, H)$ will be the set of all unitary maps $u: G \rightarrow H$, i.e. $u(1)=1$. A normalized 2-cocycle [13, Section 7] is a map $f: G \times G \rightarrow H$ such that

$$
f(1, g)=f(g, 1)=1, \quad f(g, h) f(g h, l)=f(h, l) f(g, h l)
$$

for all $g, h, l \in G$. The map $f: G \times G \rightarrow H, f(g, h):=1$, for all $g, h \in G$, is called the trivial cocycle. The set of all normalized 2-cocycles is denoted by $Z^{2}(G, H)$. Two cocycles $f$ and $f^{\prime}$ 
are called cohomologous, and we denote this by $f \approx f^{\prime}$, if there exists a unitary map $u: G \rightarrow H$ such that

$$
f\left(g, g^{\prime}\right)=u(g) u\left(g^{\prime}\right) f^{\prime}\left(g, g^{\prime}\right) u\left(g g^{\prime}\right)^{-1}
$$

for all $g, g^{\prime} \in G$. A normalized 2-cocycle $f$ is called a coboundary if $f$ is cohomologous with the trivial cocylcle, i.e. if there exists a unitary map $u: G \rightarrow H$ such that $f\left(g, g^{\prime}\right)=f_{u}\left(g, g^{\prime}\right):=$ $u(g) u\left(g^{\prime}\right) u\left(g g^{\prime}\right)^{-1}$, for all $g, g^{\prime} \in G$. " $\approx$ " is an equivalence relation on $Z^{2}(G, H)$ and we denote by $\mathcal{H}^{2}(G, H):=Z^{2}(G, H) / \approx$ the corresponding factor set. $\mathcal{H}^{2}(G, H)$ is called the second cohomology group of $G$ with coefficients in the Abelian group $H$ [1]. The following result is folklore: it describes all the normalized 2-cocycles $f: C_{n} \times C_{n} \rightarrow C_{2}$.

Proposition 2.1. Let $n$ be a positive integer. There exists a bijection $\mathcal{U}\left(C_{n}, C_{2}\right) \simeq Z^{2}\left(C_{n}, C_{2}\right)$ such that the 2-cocycle $f=f_{t}: C_{n} \times C_{n} \rightarrow C_{2}$ associated to the unitary map $t \in \mathcal{U}\left(C_{n}, C_{2}\right)$ is given by

$$
f_{t}\left(c^{i}, c^{j}\right):=\prod_{p=0}^{i-1} t\left(c^{p}\right) \prod_{q=0}^{j-1} t\left(c^{q}\right) \prod_{r=0}^{i+j-1} t\left(c^{r}\right)
$$

for all $i, j=1, \ldots, n$.

Proof. The fact that $f_{t}$ is a normalized 2-cocycle follows from a straightforward computation and moreover, $f_{t}$ can be written equivalently as

$$
f_{t}\left(c^{i}, c^{j}\right)=t\left(c^{0}\right) t\left(c^{1}\right) \cdots t\left(c^{i-1}\right) t\left(c^{j}\right) t\left(c^{j+1}\right) \cdots t\left(c^{j+i-1}\right)
$$

for all $i, j=1, \ldots, n$. The inverse of the map $t \mapsto f_{t}$ is constructed as follows: let $f: C_{n} \times C_{n} \rightarrow$ $C_{2}$ be a normalized 2-cocycle and define $t=t_{f}: C_{n} \rightarrow C_{2}, t\left(c^{j}\right):=f\left(c, c^{j}\right)$, for all $j=1, \ldots, n$. Using the cocycle condition (1) and induction on $i$ we easily obtain that (2) holds and the correspondence $\left(f \mapsto t_{f}, t \mapsto f_{t}\right)$ is bijective.

\section{Crossed products of Hopf algebras}

We shall review the construction of the crossed product of two Hopf algebras introduced in [7, Lemma 1.2.10] as a special case of the cocycle bicrossproduct [6, Theorem 2.20]. It can be also obtained as a special case of the unified product of [4, Theorem 2.4, Examples 2.5(2)]. From

now on $k$ will be an arbitrary field and we shall use $\otimes$ instead of $\otimes_{k}$. For the comultiplication of a Hopf algebra we use Sweedler's $\Sigma$-notation with suppressed summation sign: $\Delta(a)=a_{(1)} \otimes a_{(2)}$. If $A$ and $H$ are Hopf algebras and $f: H \otimes H \rightarrow A$ is a $k$-linear map, we denote $f(g, h)=f(g \otimes h)$, for all $g, h \in H$. A $k$-linear map $\triangleright: H \otimes A \rightarrow A$ is called a weak action [13] of $H$ on $A$ if for any $a, b \in A, h \in H:$

$$
\begin{aligned}
& 1_{H} \triangleright a=a, \\
& h \triangleright 1_{A}=\varepsilon_{H}(h) 1_{A}, \\
& h \triangleright(a b)=\left(h_{(1)} \triangleright a\right)\left(h_{(2)} \triangleright b\right) .
\end{aligned}
$$

Let $A$ and $H$ be two Hopf algebras, $f: H \otimes H \rightarrow A$ a $k$-linear map and $\triangleright: H \otimes A \rightarrow A$ a weak action of $H$ on $A$. We denote by $A \#_{f}^{\triangleright} H$ the $k$-vector space $A \otimes H$ endowed with the following multiplication

$$
(a \# g) \cdot(b \# h):=a\left(g_{(1)} \triangleright b\right) f\left(g_{(2)}, h_{(1)}\right) \# g_{(3)} h_{(2)}
$$


for all $a, b \in A, g, h \in H$, where \# stands for $\otimes$. The object $A \#_{f}^{\triangleright} H$ is called the crossed product of $A$ with $H$ if it is a Hopf algebra with the multiplication (6), the unit $1_{A} \# 1_{H}$ and the coalgebra structure given by the tensor product of coalgebras. [2, Proposition 1.1] and [7, Lemma 1.2.10] proves that $A \#_{f}^{\triangleright} H$ is a crossed product of $A$ with $H$ if and only if $f$ and $\triangleright$ are morphisms of coalgebras satisfying the following compatibilities

$$
\begin{aligned}
& f\left(h, 1_{H}\right)=f\left(1_{H}, h\right)=\varepsilon_{H}(h) 1_{A}, \\
& {\left[g_{(1)} \triangleright\left(h_{(1)} \triangleright a\right)\right] f\left(g_{(2)}, h_{(2)}\right)=f\left(g_{(1)}, h_{(1)}\right)\left(\left(g_{(2)} h_{(2)}\right) \triangleright a\right),} \\
& \left(g_{(1)} \triangleright f\left(h_{(1)}, l_{(1)}\right)\right) f\left(g_{(2)}, h_{(2)} l_{(2)}\right)=f\left(g_{(1)}, h_{(1)}\right) f\left(g_{(2)} h_{(2)}, l\right), \\
& g_{(1)} \otimes\left(g_{(2)} \triangleright a\right)=g_{(2)} \otimes\left(g_{(1)} \triangleright a\right), \\
& g_{(1)} h_{(1)} \otimes f\left(g_{(2)}, h_{(2)}\right)=g_{(2)} h_{(2)} \otimes f\left(g_{(1)}, h_{(1)}\right)
\end{aligned}
$$

for all $a \in A, g, h, l \in H$. Whenever (3)-(5) and (7)-(11) hold, $(A, H, \triangleright, f)$ is called a crossed system of Hopf algebras. The antipode of $A \#_{f}^{\triangleright} H$ is given for any $a \in A$ and $h \in H$ by

$$
S(a \# h):=\left(S_{A}\left[f\left(S_{H}\left(h_{(2)}\right), h_{(3)}\right)\right] \# S_{H}\left(h_{(1)}\right)\right) \cdot\left(S_{A}(a) \# 1_{H}\right) .
$$

A coalgebra split extension of $A$ by $H[2]$ is a triple $(E, i, \pi)$ consisting of a Hopf algebra $E$ and two Hopf algebra homomorphisms $i: A \rightarrow E$ and $\pi: E \rightarrow H$ such that $i$ is injective, $\pi$ has a section as a coalgebra map and $i(A) \cong E^{\operatorname{co}(H)}:=\left\{x \in E \mid x_{(1)} \otimes \pi\left(x_{(2)}\right)=x \otimes 1\right\}$.

If $E$ is finite-dimensional, then the sequence $A \stackrel{i}{\longrightarrow} E \stackrel{\pi}{\longrightarrow} H$ is an exact sequence of Hopf algebras [6]. Two coalgebra split extensions $(E, i, \pi),\left(E^{\prime}, i^{\prime}, \pi^{\prime}\right)$ of $A$ by $H$ are called equivalent if there exists an isomorphism of Hopf algebras $\psi: E \rightarrow E^{\prime}$ that stabilizes $A$ and co-stabilizes $H$, i.e. the following diagram

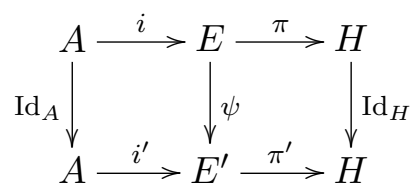

is commutative. Any crossed product $A \#_{f}^{\triangleright} H$ is a coalgebra split extension of $A$ by $H$ via $i_{A}: A \rightarrow A \#_{f}^{\triangleright} H, i_{A}(a)=a \# 1_{H}$, for all $a \in A$, and $\pi_{H}: A \#_{f}^{\triangleright} H \rightarrow H, \pi_{H}(a \# h)=\varepsilon_{A}(a) h$, for all $a \in A$ and $h \in H$. Conversely, any coalgebra split extension of $A$ by a cocommutative Hopf algebra $H$ is equivalent to a crossed product extension $\left(A \#_{f}^{\triangleright} H, i_{A}, \pi_{H}\right)$ of $A$ by $H[2$, Proposition 1.3]. More precisely, if $\varphi: H \rightarrow E$ is a unit preserving coalgebra map that splits $\pi$ then the action $\triangleright=\triangleright_{\varphi}$ and the cocycle $f=f_{\varphi}$ implemented by $\varphi$ are given by: $h \triangleright a:=$ $\varphi\left(h_{(1)}\right) a S \varphi\left(h_{(2)}\right)$ and $f(g, h):=\varphi\left(g_{(1)}\right) \varphi\left(h_{(1)}\right) S \varphi\left(g_{(2)} h_{(2)}\right)$, for all $g, h \in H$ and $a \in A$. Thus, the classification of all coalgebra split extensions of $A$ by $H$ reduces to the classification of all crossed products $A \#_{f}^{\triangleright} H$. The classifying object for all coalgebra split extensions of $A$ by $H$, denoted by $\mathcal{H}^{2}(H, A)$, was introduced in [2, Remark 2.4]. For the reader's convenience we recall briefly its construction: let $\mathcal{C S}(A, H)$ be the set of all pairs $(\triangleright, f)$ such that $(A, H, \triangleright, f)$ is a crossed system of Hopf algebras. Two pairs $(\triangleright, f)$ and $\left(\triangleright^{\prime}, f^{\prime}\right) \in \mathcal{C S}(A, H)$ are called cohomologous and we denote this by $(\triangleright, f) \approx\left(\triangleright^{\prime}, f^{\prime}\right)$ if there exists $r: H \rightarrow A$ an unitary cocentral map ${ }^{1}$ such that for any $a \in A$ and $h, g \in H$ we have

$$
\begin{aligned}
& h \triangleright^{\prime} a=r\left(h_{(1)}\right)\left(h_{(2)} \triangleright a\right)\left(S_{A} \circ r\right)\left(h_{(3)}\right), \\
& f^{\prime}(h, g)=r\left(h_{(1)}\right)\left(h_{(2)} \triangleright r\left(g_{(1)}\right)\right) f\left(h_{(3)}, g_{(2)}\right)\left(S_{A} \circ r\right)\left(h_{(4)} g_{(3)}\right) .
\end{aligned}
$$

Then [4, Theorem 3.4] proves that $(\triangleright, f) \approx\left(\triangleright^{\prime}, f^{\prime}\right)$ if and only if there exists a Hopf algebra isomorphism $A \#_{f}^{\triangleright} H \cong A \#_{f^{\prime}}^{\triangleright^{\prime}} H$ that stabilizes $A$ and co-stabilizes $H$. Thus, $\approx$ is an

\footnotetext{
${ }^{1}$ I.e. $r$ is a unit preserving coalgebra map such that $r\left(h_{(1)}\right) \otimes h_{(2)}=r\left(h_{(2)}\right) \otimes h_{(1)}$, for all $h \in H$.
} 
equivalence relation on the set $\mathcal{C S}(A, H)$ and $\mathcal{H}^{2}(H, A)$ is the pointed quotient set defined by $\mathcal{H}^{2}(H, A):=\mathcal{C S}(A, H) / \approx$. Now, if $H$ is cocommutative and $A$ commutative then $\mathcal{H}^{2}(H, A)$ coincides with the second cohomological group as constructed by Sweedler [14]; however, for arbitrary Hopf algebras $A$ and $H$, we could not find a complex for which $\mathcal{H}^{2}(H, A)$ is the associated cohomological group. We denote by $\operatorname{CRP}(H, A)$ the set of types of Hopf algebra isomorphisms of all crossed products $A \#_{f}^{\triangleright} H$ associated to all crossed systems $(A, H, \triangleright, f)$. Two equivalent extensions are isomorphic and hence there exists a canonical surjection $\mathcal{H}^{2}(H, A) \rightarrow \mathrm{CRP}(H, A)$. As in the case of groups, it turns out that the two classifying objects $\mathcal{H}^{2}(H, A)$ and $\operatorname{CRP}(H, A)$ are different: [2, Proposition 4.2] proves that for the pair of Hopf algebras $(H, A):=\left(H_{4}, k[Y]\right)$ we have that $\operatorname{CRP}(H, A) \cong C_{2}$ while $\mathcal{H}^{2}(H, A) \cong k$, where $k$ is the base field.

\section{The classification of coalgebra split extensions of $H_{4}$ by $k\left[C_{n}\right]$}

Let $k$ be a field of characteristic $\neq 2, k\left[C_{n}\right]$ the group Hopf algebra of the cyclic group $C_{n}$ of order $n$ generated by $c$ and let $H_{4}$ be Sweedler's 4-dimensional Hopf algebra generated by the group-like element $g$ and the $(1, g)$-primitive element $x$ (that is $\Delta(x)=x \otimes 1+g \otimes x)$ subject to the following relations

$$
g^{2}=1, \quad x^{2}=0, \quad x g=-g x .
$$

We classify all coalgebra split extensions of $H_{4}$ by $k\left[C_{n}\right]$ following the strategy presented in the introduction: first, we describe explicitly all crossed systems $\left(H_{4}, k\left[C_{n}\right], \triangleright, f\right)$, then we describe by generators and relations the associated crossed products and, finally, we classify such extensions by computing the classifying objects $\mathcal{H}^{2}\left(k\left[C_{n}\right], H_{4}\right)$ and $\operatorname{CRP}\left(k\left[C_{n}\right], H_{4}\right)$. To any unitary map $t: C_{n} \rightarrow C_{2}$ we associate the function $\sigma_{t}: \mathbb{N} \rightarrow \mathbb{N}$ defined by $\sigma_{t}(0)=\sigma_{t}(1):=0$ and for any positive integer $j \geq 2$

$$
\sigma_{t}(j):=\left\{\begin{array}{lll}
1+\operatorname{ord} t(c)+\cdots+\operatorname{ord} t\left(c^{j-1}\right) & \text { if } & 2 \mid j, \\
\operatorname{ord} t(c)+\cdots+\operatorname{ord} t\left(c^{j-1}\right) & \text { if } & 2 \nmid j .
\end{array}\right.
$$

The following gives the parametrization of all crossed systems $\left(H_{4}, k\left[C_{n}\right], \triangleright, f\right)$.

Theorem 3.1. Let $k$ be a field of characteristic $\neq 2$ and $n$ a positive integer. Then there exists a bijective correspondence between the set of all crossed systems of Hopf algebras $\left(H_{4}, k\left[C_{n}\right], \triangleright, f\right)$ and the set $\mathcal{C S}(n, k) \subseteq \mathcal{U}\left(C_{n}, C_{2}\right) \times k^{*}$ consisting of all pairs $(t, \lambda)$, where $t: C_{n} \rightarrow C_{2}$ is a unitary map and $\lambda \in k^{*}$ is such that $\lambda^{n}=(-1)^{\sigma_{t}(n)}$.

Under this bijection the crossed system $\left(H_{4}, k\left[C_{n}\right], \triangleright, f\right)$ corresponding to $(t, \lambda)$ is given by

$$
\begin{aligned}
& c^{j} \triangleright 1=1, \quad c^{j} \triangleright g=g, \quad c^{j} \triangleright x=(-1)^{\sigma_{t}(j)} \lambda^{j} x, \quad c^{j} \triangleright(g x)=(-1)^{\sigma_{t}(j)} \lambda^{j} g x, \\
& f\left(c^{i}, c^{j}\right)=\prod_{p=0}^{i-1} t\left(c^{p}\right) \prod_{q=0}^{j-1} t\left(c^{q}\right) \prod_{r=0}^{i+j-1} t\left(c^{r}\right)
\end{aligned}
$$

for all $i, j=1, \ldots, n$.

Proof. We have to describe all pairs $(\triangleright, f)$ of coalgebra maps $\triangleright: k\left[C_{n}\right] \otimes H_{4} \rightarrow H_{4}$ and $f: k\left[C_{n}\right] \otimes k\left[C_{n}\right] \rightarrow H_{4}$ that satisfy the compatibility conditions (3)-(5) and (7)-(11). For such a pair, the compatibilities (10)-(11) hold since $k\left[C_{n}\right]$ is cocommutative.

Let $(\triangleright, f)$ be a pair such that $\left(H_{4}, k\left[C_{n}\right], \triangleright, f\right)$ is a crossed system. We will first prove that $f\left(c^{i}, c^{j}\right) \in\{1, g\}$, for all $i, j=1, \ldots, n$, the corresponding map $f: C_{n} \times C_{n} \rightarrow C_{2}=\{1, g\}$ is a classical normalized 2-cocycle of groups in the sense of (1) and the action $\triangleright: k\left[C_{n}\right] \otimes H_{4} \rightarrow H_{4}$ is given by

$$
c^{j} \triangleright 1=1, \quad c^{j} \triangleright g=g, \quad c^{j} \triangleright x=\lambda_{j} x, \quad c^{j} \triangleright(g x)=\lambda_{j} g x
$$


for all $j=1, \ldots, n$, where the scalars $\lambda_{j} \in k$ are such that (14) holds, which in our case takes the equivalent form

$$
\left(c^{i} \triangleright\left(c^{j} \triangleright a\right)\right) f\left(c^{i}, c^{j}\right)=f\left(c^{i}, c^{j}\right)\left(c^{i+j} \triangleright a\right)
$$

for all $i, j=1, \ldots, n$ and $a \in H_{4}$. In the next step we will use Proposition 2.1: any classical normalized 2-cocycle $f: C_{n} \times C_{n} \rightarrow C_{2}$ is implemented by a unique unitary map $t: C_{n} \rightarrow C_{2}$ such that (13) holds. Finally, using this description of $f$, we will prove that (15) holds if and only if $\lambda_{j}=(-1)^{\sigma_{t}(j)} \lambda_{1}^{j}$, for all $j=1, \ldots, n$, and $\lambda_{1}^{n}=(-1)^{\sigma_{t}(n)}$. This will finish the proof.

First, since $f: k\left[C_{n}\right] \otimes k\left[C_{n}\right] \rightarrow H_{4}$ is a coalgebra map, $f\left(c^{i}, c^{j}\right)$ is a grouplike element in $H_{4}$. Therefore $f\left(c^{i}, c^{j}\right) \in\{1, g\}$, for all $i, j=1, \ldots, n$. Hence, $f$ is uniquely determined by its restriction to $C_{n}$, which we will also denote by $f: C_{n} \times C_{n} \rightarrow C_{2}$. Similarly, as $\triangleright$ : $k\left[C_{n}\right] \otimes H_{4} \rightarrow H_{4}$ is a coalgebra map, we obtain that $c^{j} \triangleright g \in\{1, g\}$, for all $j=1, \ldots, n$. We claim that $c^{j} \triangleright g=g$, for all $j=1, \ldots, n$. Indeed, assume that $c^{j} \triangleright g=1$, for some $j=1, \ldots, n$. Applying (15) for $i:=n-j$ and $a:=g$ and taking into account the normalizing condition (7), we obtain $f\left(c^{n-j}, c^{j}\right)=f\left(c^{n-j}, c^{j}\right) g$, which gives a contradiction as $\operatorname{Im}(f) \subseteq\{1, g\}$ and $g^{2}=1$. Therefore, $c^{j} \triangleright g=g$, for all $j=1, \ldots, n$.

Using once again that $\triangleright$ is a coalgebra map and the fact that $x$ is a $(1, g)$-primitive element of $H_{4}$ we obtain that $c^{j} \triangleright x$ is also an $(1, g)$-primitive element of $H_{4}$. Hence, using [3, Lemma 4.2], we obtain that $c^{j} \triangleright x=\mu_{j}-\mu_{j} g+\lambda_{j} x$, for some scalars $\mu_{j}, \lambda_{j} \in k$. From this formula of $c^{j} \triangleright x$ and the compatibility condition (5) we obtain

$$
0=c^{j} \triangleright\left(x^{2}\right)=\left(c^{j} \triangleright x\right)\left(c^{j} \triangleright x\right)=2 \mu_{j}^{2}-2 \mu_{j}^{2} g+2 \mu_{j} \lambda_{j} x .
$$

Thus $\mu_{j}=0$ and $c^{j} \triangleright x=\lambda_{j} x$, for all $j=1, \ldots, n$. Applying (5) once again we obtain: $c^{j} \triangleright(g x)=\left(c^{j} \triangleright g\right)\left(c^{j} \triangleright x\right)=\lambda_{j} g x$. Thus, the formula (14) holds. Now, the compatibility condition (9) is equivalent to

$$
\left(c^{i} \triangleright f\left(c^{j}, c^{k}\right)\right) f\left(c^{i}, c^{j+k}\right)=f\left(c^{i}, c^{j}\right) f\left(c^{i+j}, c^{k}\right)
$$

for all $i, j, k=1, \ldots, n$. Since $c^{i} \triangleright f\left(c^{j}, c^{k}\right)=f\left(c^{j}, c^{k}\right)$, for all $i, j$ and $k$, the condition (9) is, thus, equivalent to the fact that $f: C_{n} \times C_{n} \rightarrow C_{2}$ is a usual normalized 2-cocycle for groups in the sense of (1). It follows then from Proposition 2.1 that there exists a unique map $t: C_{n} \rightarrow C_{2}$ such that $t(1)=1$ and (13) holds for all $i, j=1, \ldots, n$. In particular, $f\left(c, c^{j}\right)=t\left(c^{j}\right)$, for all $j=1, \ldots, n$.

With the information collected so far on $f$ and $\triangleright$ we turn now to the compatibility condition (15) and see when it is satisfied: this is in fact the last compatibility that needs to be fulfilled in order for $\left(H_{4}, k\left[C_{n}\right], \triangleright, f\right)$ to be a crossed system of Hopf algebras. We notice that (15) holds automatically if $a=g$, since $c^{i} \triangleright g=g$, for all $i=1, \ldots, n$. On the other hand, using (15) for $a=x$ and $c^{i}=c^{j}=c$ we obtain

$$
\lambda_{1}^{2} x f(c, c)=f(c, c) \lambda_{2} x .
$$

Multiplying to the left by $f(c, c)=t(c) \in\{1, g\}$ we obtain

$$
\lambda_{2} x=\lambda_{1}^{2} f(c, c) x f(c, c)=(-1)^{1+o r d} f(c, c) \lambda_{1}^{2} x .
$$

Thus, $\lambda_{2}=(-1)^{\sigma_{t}(2)} \lambda_{1}^{2}$. By writing (15) for $\left(c, c^{2}\right)$ and $a=x$ we obtain that $\lambda_{1} \lambda_{2} x f\left(c, c^{2}\right)=$ $f\left(c, c^{2}\right) \lambda_{3} x$, from which we deduce

$$
\lambda_{3} x=\lambda_{1} \lambda_{2} f\left(c, c^{2}\right) x f\left(c, c^{2}\right)=(-1)^{\operatorname{ord} f(c, c)+\operatorname{ord} f\left(c, c^{2}\right)} \lambda_{1}^{3} x .
$$


Thus, $\lambda_{3}=(-1)^{\sigma_{t}(3)} \lambda_{1}^{3}$. By induction, it follows that $\lambda_{j}=(-1)^{\sigma_{t}(j)} \lambda_{1}^{j}$, for all $j=2, \ldots, n-1$. Applying (15) for $\left(c, c^{n-1}\right)$ and $a=x$, we obtain that $\lambda_{1} \lambda_{n-1} x f\left(c, c^{n-1}\right)=f\left(c, c^{n-1}\right) x$, which is equivalent to

$$
x=\lambda_{1} \lambda_{n-1} f\left(c, c^{n-1}\right) x f\left(c, c^{n-1}\right)=(-1)^{\sigma_{t}(n)} \lambda_{1}^{n} x .
$$

Thus, $\lambda_{1}^{n}=(-1)^{\sigma_{t}(n)}$, as needed.

Conversely, let $\lambda \in k$ be such that $\lambda^{n}=(-1)^{\sigma_{t}(n)}$ and define $\lambda_{j}:=(-1)^{\sigma_{t}(j)} \lambda^{j}$. Then we can prove that (15) holds. Indeed, it suffices to see that (15) holds for $a=x$. Observe first that, since $(-1)^{\sigma_{t}(n+j)}=(-1)^{\sigma_{t}(n)+\sigma_{t}(j)}$, we have $c^{n+j} \triangleright x=(-1)^{\sigma_{t}(n+j)} \lambda^{n+j} x$, and thus, $c^{j} \triangleright x=(-1)^{\sigma_{t}(j)} \lambda^{j} x$, for all non-negative integers $j$. Secondly,

$$
\begin{aligned}
x t(c) t\left(c^{2}\right) \cdots t\left(c^{j-1}\right) & =(-1)^{1+\operatorname{ord} t(c)} t(c) x t\left(c^{2}\right) \cdots t\left(c^{j-1}\right)=\cdots \\
& =(-1)^{j-1+\operatorname{ord} t(c)+\cdots+\operatorname{ord} t\left(c^{j-1}\right)} t(c) t\left(c^{2}\right) \cdots t\left(c^{j-1}\right) x \\
& =(-1)^{\sigma t(j)} t(c) t\left(c^{2}\right) \cdots t\left(c^{j-1}\right) x .
\end{aligned}
$$

Using this formula, we have

$$
\begin{aligned}
\left(c^{i} \triangleright\left(c^{j} \triangleright x\right)\right) f\left(c^{i}, c^{j}\right) & =(-1)^{\sigma_{t}(i)+\sigma_{t}(j)} \lambda^{i+j} x f\left(c^{i}, c^{j}\right) \\
& =(-1)^{\sigma_{t}(i)+\sigma_{t}(j)} \lambda^{i+j} x \prod_{p=0}^{i-1} t\left(c^{p}\right) \prod_{q=0}^{j-1} t\left(c^{q}\right) \prod_{r=0}^{i+j-1} t\left(c^{r}\right) \\
& =(-1)^{2 \sigma_{t}(i)+2 \sigma_{t}(j)+\sigma_{t}(i+j)} \lambda^{i+j} f\left(c^{i}, c^{j}\right) x \\
& =(-1)^{\sigma_{t}(i+j)} \lambda^{i+j} f\left(c^{i}, c^{j}\right) x=f\left(c^{i}, c^{j}\right)\left(c^{i+j} \triangleright x\right),
\end{aligned}
$$

which proves our assertion and completes the proof.

Remark 3.2. If $t_{1} \in \mathcal{U}\left(C_{n}, C_{2}\right)$ is the trivial map, i.e. $t_{1}\left(c^{i}\right)=1$, for any $i=1, \ldots, n-1$, then $2 \mid \sigma_{t_{1}}(j)$, for all $j \geq 0$. Then the crossed systems $\left(H_{4}, k\left[C_{n}\right], \triangleright, f\right)$ corresponding to $\left(t_{1}, \lambda\right)$, $\lambda^{n}=1$, are precisely the matched pairs of [3, Proposition 4.3].

Next we describe by generators and relations all crossed products $H_{4} \# k\left[C_{n}\right]$ associated to the crossed systems from Theorem 3.1. We need to introduce the following notation: for any $i, j=1, \ldots, n-1$ we shall denote by $j \star i$ the following number

$$
j \star i= \begin{cases}j+i & \text { if } j+i \leq n, \\ j+i-n & \text { if } j+i>n .\end{cases}
$$

Corollary 3.3. Let $k$ be a field of characteristic $\neq 2$ and $n$ a positive integer. Then $H_{4} \#_{f}^{\triangleright} k\left[C_{n}\right] \cong$ $H_{4 n, \lambda, t}$, for some $(t, \lambda) \in \mathcal{C S}(n, k)$, where we denote by $H_{4 n, \lambda, t}$ the $4 n$-dimensional Hopf algebra having $\left\{d_{i}, g d_{i}, x d_{i}, g x d_{i} \mid i=1, \ldots, n\right\}$ as a $k$-basis, the unit $1=d_{n}$ and the multiplication is subject to the following relations

$$
\begin{aligned}
& g^{2}=1, \quad x^{2}=0, \quad x g=-g x, \quad d_{i} g=g d_{i}, \\
& d_{i} x=(-1)^{\sigma_{t}(i)} \lambda^{i} x d_{i}, \quad d_{j} d_{i}=t(c) \cdots t\left(c^{j-1}\right) t\left(c^{i}\right) t\left(c^{i+1}\right) \cdots t\left(c^{i+j-1}\right) d_{j \star i}
\end{aligned}
$$

for all $i, j=1, \ldots, n-1$. The coalgebra structure and the antipode on $H_{4 n, \lambda, t}$ are given by

$$
\begin{aligned}
& \Delta(g)=g \otimes g, \quad \Delta\left(d_{i}\right)=d_{i} \otimes d_{i}, \quad \Delta(x)=x \otimes 1+g \otimes x, \quad \varepsilon(g)=\varepsilon\left(d_{i}\right)=1, \\
& \varepsilon(x)=0, \quad S(g)=g, \quad S(x)=-g x, \quad S\left(d_{i}\right)=d_{n-i} \prod_{p=0}^{i-1} t\left(c^{p}\right) t\left(c^{n-1-p}\right)
\end{aligned}
$$

for all $i=1, \ldots, n-1$. 
Proof. The Hopf algebra $H_{4 n, \lambda, t}$ is the crossed product $H_{4} \#_{f}^{\triangleright} k\left[C_{n}\right]$ associated to the pair $(t, \lambda)$ as in Theorem 3.1. Up to canonical identification, the crossed product $H_{4} \#{ }_{f}^{\triangleright} k\left[C_{n}\right]$ is generated as an algebra by $g=g \# 1, x=x \# 1$ and $d_{i}=1 \# c^{i}$ for $i \in\{1, \ldots, n\}$. As $H_{4}$ is a Hopf subalgebra of $H_{4 n, \lambda, t}$, the relations $g^{2}=1, x^{2}=0$ and $g x=-x g$ also hold in $H_{4 n, \lambda, t}$. Now, in the crossed product $H_{4} \#_{f}^{\triangleright} k\left[C_{n}\right]$ the following relations hold

$$
\begin{aligned}
& d_{i} g=\left(1 \# c^{i}\right)(g \# 1)=c^{i} \triangleright g \# c^{i}=g \# c^{i}=(g \# 1)\left(1 \# c^{i}\right)=g d_{i}, \\
& d_{i} x=\left(1 \# c^{i}\right)(x \# 1)=c^{i} \triangleright x \# c^{i}=(-1)^{\sigma_{t}(i)} \lambda^{i} x \# c^{i}=(-1)^{\sigma_{t}(i)} \lambda^{i} x d_{i}, \\
& d_{j} d_{i}=\left(1 \# c^{j}\right)\left(1 \# c^{i}\right)=f\left(c^{j}, c^{i}\right) \# c^{j+i}=t(c) \cdots t\left(c^{j-1}\right) t\left(c^{i}\right) t\left(c^{i+1}\right) \cdots t\left(c^{i+j-1}\right) d_{j \star i}
\end{aligned}
$$

for all $i, j=1, \ldots, n-1$. The formula for the antipode follows from (12).

Next we shall give necessary and sufficient conditions for two Hopf algebras $H_{4 n, \lambda, t}$ and $H_{4 n, \lambda^{\prime}, t^{\prime}}$ to be isomorphic. We recall from [3, Lemma 4.6] that $\operatorname{Aut}_{\text {Hopf }}\left(H_{4}\right) \cong k^{*}$ : explicitly, any automorphism $u: H_{4} \rightarrow H_{4}$ is of the form

$$
u(g)=g, \quad u(x)=\beta x, \quad u(g x)=\beta g x
$$

for some non-zero scalar $\beta \in k^{*}$. It what follows, the automorphism $u$ of $H_{4}$ implemented by $\beta \in k^{*}$ as in (16) will be denoted by $u=u_{\beta}$.

Theorem 3.4. Let $k$ be a field of characteristic $\neq 2, n$ a positive integer and $(t, \lambda),\left(t^{\prime}, \lambda^{\prime}\right) \in$ $\mathcal{C S}(n, k)$. Then there is a bijective correspondence between the set of all Hopf algebra isomorphisms $\psi: H_{4 n, \lambda, t} \rightarrow H_{4 n, \lambda^{\prime}, t^{\prime}}$ and the set of all triples $(\beta, r, s) \in k^{*} \times \mathcal{U}\left(C_{n}, C_{2}\right) \times\{s \in\{1, \ldots, n-$ $1\} \mid \operatorname{gcd}(s, n)=1\}$ satisfying the following compatibility conditions for any $i, j=1, \ldots, n$

$$
\begin{aligned}
& \prod_{p=0}^{i-1} t\left(c^{p}\right) t\left(c^{p+j}\right)=r\left(c^{i}\right) r\left(c^{j}\right) r\left(c^{i+j}\right) \prod_{q=0}^{i s-1} t^{\prime}\left(c^{q}\right) t^{\prime}\left(c^{q+j s}\right), \\
& (-1)^{1+\sigma_{t}(i)+\operatorname{ord} r\left(c^{i}\right)} \lambda^{i} \beta=(-1)^{\sigma_{t^{\prime}}(i s)}\left(\lambda^{\prime}\right)^{i s} .
\end{aligned}
$$

Under the above bijection the isomorphism $\psi=\psi_{(\beta, r, s)}: H_{4 n, \lambda, t} \rightarrow H_{4 n, \lambda^{\prime}, t^{\prime}}$ corresponding to $(\beta, r, s)$ is given by

$$
\psi\left(a \# c^{i}\right)=u_{\beta}(a) r\left(c^{i}\right) \# c^{i s}
$$

for all $a \in H_{4}$ and $i=1, \ldots, n$.

Proof. Let $\left(H_{4}, k\left[C_{n}\right], \triangleright, f_{t}\right)$ and $\left(H_{4}, k\left[C_{n}\right], \triangleright^{\prime}, f_{t^{\prime}}\right)$ be the crossed systems corresponding to $(t, \lambda)$ and respectively $\left(t^{\prime}, \lambda^{\prime}\right)$ given in Theorem 3.1. Then $H_{4 n, \lambda, t}=H_{4} \#_{f_{t}}^{\triangleright} k\left[C_{n}\right]$ and $H_{4 n, \lambda^{\prime}, t^{\prime}}=$ $H_{4} \#_{f_{t^{\prime}}^{\prime}}^{\triangleright^{\prime}} k\left[C_{n}\right]$. By [2, Theorem 2.1], the set of all Hopf algebra morphisms $\psi: H_{4 n, \lambda, t} \rightarrow H_{4 n, \lambda^{\prime}, t^{\prime}}$ is in bijective correspondence with the set of all quadruples $(u, p, r, v)$, where $p: H_{4} \rightarrow k\left[C_{n}\right]$ is a Hopf algebra map, $u: H_{4} \rightarrow H_{4}, r: k\left[C_{n}\right] \rightarrow H_{4}$ and $v: k\left[C_{n}\right] \rightarrow k\left[C_{n}\right]$ are unitary coalgebra maps satisfying the following compatibility conditions:

$(\mathrm{CP} 1) \quad u\left(a_{(1)}\right) \otimes p\left(a_{(2)}\right)=u\left(a_{(2)}\right) \otimes p\left(a_{(1)}\right)$,

$(\mathrm{CP} 2) \quad r\left(h_{(1)}\right) \otimes v\left(h_{(2)}\right)=r\left(h_{(2)}\right) \otimes v\left(h_{(1)}\right)$,

(CP3) $u(a b)=u\left(a_{(1)}\right)\left(p\left(a_{(2)}\right) \triangleright^{\prime} u\left(b_{(1)}\right)\right) f^{\prime}\left(p\left(a_{(3)}\right), p\left(b_{(2)}\right)\right)$,

(CP4) $v(h) v(g)=p\left(f\left(h_{(1)}, g_{(1)}\right)\right) v\left(h_{(2)} g_{(2)}\right)$,

(CP5) $v(h) p(a)=p\left(h_{(1)} \triangleright a\right) v\left(h_{(2)}\right)$,

(CP6) $r\left(h_{(1)}\right)\left(v\left(h_{(2)}\right) \triangleright^{\prime} r\left(g_{(1)}\right)\right) f^{\prime}\left(v\left(h_{(3)}\right), v\left(g_{(2)}\right)\right)$ 


$$
\begin{gathered}
=u\left(f\left(h_{(1)}, g_{(1)}\right)\right)\left(p\left(f\left(h_{(2)}, g_{(2)}\right) \triangleright^{\prime} r\left(h_{(4)} g_{(4)}\right)\right) f^{\prime}\left(p\left(f\left(h_{(3)}, g_{(3)}\right), v\left(h_{(5)} g_{(5)}\right)\right)\right),\right. \\
(\mathrm{CP} 7) \quad r\left(h_{(1)}\right)\left(v\left(h_{(2)}\right) \triangleright^{\prime} u\left(a_{(1)}\right)\right) f^{\prime}\left(v\left(h_{(3)}\right), p\left(a_{(2)}\right)\right) \\
=u\left(h_{(1)} \triangleright a_{(1)}\right)\left(p\left(h_{(2)} \triangleright a_{(2)}\right) \triangleright^{\prime} r\left(h_{(4)}\right)\right) f^{\prime}\left(p\left(h_{(3)} \triangleright a_{(3)}\right), v\left(h_{(5)}\right)\right)
\end{gathered}
$$

for all $a, b \in H_{4}, g, h \in k\left[C_{n}\right]$. The correspondence is such that the morphism $\psi=\psi_{(u, p, r, v)}$ associated to $(u, p, r, v)$ is given by

$$
\psi(a \# h)=u\left(a_{(1)}\right)\left(p\left(a_{(2)}\right) \triangleright^{\prime} r\left(h_{(1)}\right)\right) f^{\prime}\left(p\left(a_{(3)}\right), v\left(h_{(2)}\right)\right) \#^{\prime} p\left(a_{(4)}\right) v\left(h_{(3)}\right)
$$

for all $a \in H_{4}$ and all $h \in k\left[C_{n}\right]$. We will show that, under this bijection, isomorphisms correspond precisely to quadruples $(u, p, r, v)$, where $p: H_{4} \rightarrow k\left[C_{n}\right]$ is the trivial morphism, $u: H_{4} \rightarrow H_{4}$ and $v: k\left[C_{n}\right] \rightarrow k\left[C_{n}\right]$ are Hopf algebra automorphisms and $r: k\left[C_{n}\right] \rightarrow H_{4}$ is a unitary coalgebra map such that the following two conditions are satisfied

$$
\begin{aligned}
& f_{t}\left(c^{i}, c^{j}\right)=r\left(c^{i}\right) r\left(c^{j}\right) r\left(c^{i+j}\right) f_{t^{\prime}}\left(v\left(c^{i}\right), v\left(c^{j}\right)\right), \\
& u\left(c^{i} \triangleright a\right) r\left(c^{i}\right)=r\left(c^{i}\right)\left(v\left(c^{i}\right) \triangleright^{\prime} u(a)\right)
\end{aligned}
$$

for all $i=1, \ldots, n$ and $a \in H_{4}$. At the end we will see that such quadruples are in bijection with triples $(\beta, r, s) \in k^{*} \times \mathcal{U}\left(C_{n}, C_{2}\right) \times\{s \in\{1, \ldots, n-1\} \mid \operatorname{gcd}(s, n)=1\}$ such that (17) and (18) are satisfied.

Suppose first that $\psi=\psi_{(u, p, r, v)}: H_{4} \#_{f_{t}}^{\triangleright} k\left[C_{n}\right] \rightarrow H_{4} \#_{f_{t^{\prime}}}^{\triangleright^{\prime}} k\left[C_{n}\right]$ is an isomorphism corresponding to $(u, p, r, v)$. Thus $p: H_{4} \rightarrow k\left[C_{n}\right]$ is a Hopf algebra map; it follows from [3, Lemma 4.6] that $p(x)=0$ and $p(g x)=0$. In particular, we have

$$
\psi(x \# 1)=u(x) \# p(1)+u(g) \# p(x)=u(x) \# 1 .
$$

As $\psi$ is an isomorphism, $u(x)$ must be non-zero. Looking at (CP1) for $a=x$ and taking into account that $p(x)=0$ and $u(x) \neq 0$ we obtain $p(g)=1$. Thus, $p: H_{4} \rightarrow k\left[C_{n}\right]$ is the trivial morphism: $p(z)=\varepsilon(z) 1$, for all $z \in H_{4}$. Thus, the compatibility conditions (CP1) and (CP5) hold automatically while (CP2) holds since $k\left[C_{n}\right]$ is cocommutative. Moreover, (CP3) is equivalent to the fact that $u: H_{4} \rightarrow H_{4}$ is a Hopf algebra morphism and (CP4) is equivalent to $v: k\left[C_{n}\right] \rightarrow k\left[C_{n}\right]$ being a Hopf algebra morphism. Since $u(x) \neq 0$, it follows from [3, Lemma 4.6] that $u: H_{4} \rightarrow H_{4}$ is an automorphism of $H_{4}$. Using [2, Corollary 2.2] we obtain that $v: k\left[C_{n}\right] \rightarrow k\left[C_{n}\right]$ is also an automorphism of $k\left[C_{n}\right]$.

It remains to show that (19) and (20) hold. We claim that, in fact, these are exactly (CP6) and (CP7) written in an equivalent form. Indeed, taking into account that $p$ is the trivial map, we have that (CP7) is equivalent to the compatibility condition (20), while (CP6) is equivalent to

$$
r\left(c^{i}\right)\left(v\left(c^{i}\right) \triangleright^{\prime} r\left(c^{j}\right)\right) f_{t^{\prime}}\left(v\left(c^{i}\right), v\left(c^{j}\right)\right)=u\left(f_{t}\left(c^{i}, c^{j}\right)\right) r\left(c^{i+j}\right)
$$

for all $i, j \in\{1, \ldots, n\}$. Now $r: k\left[C_{n}\right] \rightarrow H_{4}$ is a coalgebra map, hence $r\left(c^{i}\right) \in\{1, g\}$, for all $i$. Since $v\left(c^{i}\right) \in C_{n}$ and the elements of $C_{n}$ act, via $\triangleright^{\prime}$, trivially on $\{1, g\}$, we have $v\left(c^{i}\right) \triangleright^{\prime} r\left(c^{j}\right)=$ $r\left(c^{j}\right)$, for all $i$ and $j$. Furthermore, $f_{t}\left(c^{i}, c^{j}\right) \in\{1, g\}$ for all $i$ and $j$, and $u(1)=1$ and $u(g)=g$, hence, $u\left(f\left(c^{i}, c^{j}\right)\right)=f\left(c^{i}, c^{j}\right)$, for all $i$ and $j$. These remarks show that (CP7) is equivalent to

$$
r\left(c^{i}\right) r\left(c^{j}\right) f_{t^{\prime}}\left(v\left(c^{i}\right), v\left(c^{j}\right)\right)=f_{t}\left(c^{i}, c^{j}\right) r\left(c^{i+j}\right)
$$

for all $i, j=1, \ldots, n$, which is equivalent to (19).

Conversely, let $(u, p, r, v)$ be a quadruple with $p: H_{4} \rightarrow k\left[C_{n}\right]$ the trivial morphism, $u: H_{4} \rightarrow$ $H_{4}$ and $v: k\left[C_{n}\right] \rightarrow k\left[C_{n}\right]$ Hopf algebra automorphisms and $r: k\left[C_{n}\right] \rightarrow H_{4}$ a unitary coalgebra map satisfying (19) and (20). We will prove that the compatibility conditions (CP1)-(CP7) are satisfied. Indeed, (CP1)-(CP5) are trivially fulfilled. (CP6) and (CP7) are equivalent, 
as we have seen, with (19) and (20). Thus, $(u, p, r, v)$ determines a Hopf algebra morphism $\psi: H_{4} \#_{f_{t}}^{\triangleright} k\left[C_{n}\right] \rightarrow H_{4} \#_{f_{t^{\prime}}}^{\triangleright^{\prime}} k\left[C_{n}\right]$, given by

$$
\psi(a \# h)=u(a) r\left(h_{(1)}\right) \#^{\prime} v\left(h_{(2)}\right)
$$

for all $a \in H_{4}$ and $h \in k\left[C_{n}\right]$. Since $u$ and $v$ are isomorphisms it follows from [2, Corollary 2.2] that $\psi$ is an isomorphism.

To conclude, we have established a bijective correspondence between the set of all Hopf algebra isomorphisms $\psi: H_{4 n, \lambda, t} \rightarrow H_{4 n, \lambda^{\prime}, t^{\prime}}$ and the set of all quadruples $(u, p, r, v)$ consisting of the trivial morphism $p: H_{4} \rightarrow k\left[C_{n}\right]$, two Hopf algebra automorphisms, $u: H_{4} \rightarrow H_{4}$ and $v: k\left[C_{n}\right] \rightarrow k\left[C_{n}\right]$, and a unitary coalgebra map $r: k\left[C_{n}\right] \rightarrow H_{4}$ that satisfy (19) and (20). These quadruples are, in turn, in bijection with the set of all triples $(\beta, r, s) \in k^{*} \times \mathcal{U}\left(C_{n}, C_{2}\right) \times\{s \in$ $\{1, \ldots, n-1\} \mid \operatorname{gcd}(s, n)=1\}$ such that the compatibility conditions (17) and (18) are fulfilled. Indeed, for any $u \in \operatorname{Aut}_{\text {Hopf }}\left(H_{4}\right)$ there exists a unique $\beta \in k^{*}$ such that $u=u_{\beta}$ and for any $v \in \operatorname{Aut}_{\text {Hopf }}\left(k\left[C_{n}\right]\right)$ there exists a unique $s \in\{1, \ldots, n-1\}, \operatorname{gcd}(s, n)=1$, such that $v\left(c^{i}\right)=c^{i s}$, for all $i=0, \ldots, n-1$. Furthermore, any unitary coalgebra map $r: k\left[C_{n}\right] \rightarrow H_{4}$ is uniquely determined by a unitary map $C_{n} \rightarrow C_{2}=\{1, g\}$ which we still denote by $r$. Therefore (17) and (18) are nothing but (19) and (20) in terms of $t, t^{\prime}, \beta, r$ and $s$.

Remark 3.5. Although condition (17) is given in terms of $t, t^{\prime}, r$ and $s$, in practice it is rather difficult to work with. In such a situation, it is more convenient to consider the equivalent condition (19) which says that the normalized 2-cocycles $f_{t}$ and $f_{t^{\prime}} \circ(v \times v)$, where $v$ is the automorphism of $C_{n}$ associated to $s$, are cohomologous and that the coboundary by which they differ is the one associated to $r$.

The main result of the paper is the following theorem:

Theorem 3.6. Let $k$ be a field of characteristic $\neq 2$ and $n$ a positive integer. Then:

1. There exists a bijection between $\mathcal{H}^{2}\left(k\left[C_{n}\right], H_{4}\right)$ and the quotient set $\mathcal{C S}(n, k) / \approx$, where $\approx$ is the equivalence relation on $\mathcal{C S}(n, k)$ defined by: $(t, \lambda) \approx\left(t^{\prime}, \lambda^{\prime}\right)$ if and only if there exists $r \in \mathcal{U}\left(C_{n}, C_{2}\right)$ such that for all $i, j=1, \ldots, n$

$$
\begin{aligned}
& \prod_{p=0}^{i-1} t\left(c^{p}\right) t\left(c^{p+j}\right)=r\left(c^{i}\right) r\left(c^{j}\right) r\left(c^{i+j}\right) \prod_{q=0}^{i-1} t^{\prime}\left(c^{q}\right) t^{\prime}\left(c^{q+j}\right), \\
& (-1)^{1+\sigma_{t}(i)+\operatorname{ord} r\left(c^{i}\right)} \lambda^{i}=(-1)^{\sigma_{t^{\prime}}(i)}\left(\lambda^{\prime}\right)^{i} .
\end{aligned}
$$

2. There exists a bijection between $\operatorname{CRP}\left(k\left[C_{n}\right], H_{4}\right)$ and the quotient set $\mathcal{C S}(n, k) / \equiv$, where $\equiv$ is the equivalence relation on $\mathcal{C S}(n, k)$ defined by: $(t, \lambda) \equiv\left(t^{\prime}, \lambda^{\prime}\right)$ if and only if there exists $(\beta, r, s) \in k^{*} \times \mathcal{U}\left(C_{n}, C_{2}\right) \times\{s \in\{1, \ldots, n-1\} \mid \operatorname{gcd}(s, n)=1\}$ satisfying the compatibility conditions (17) and (18).

Proof. It follows from Theorems 3.1 and 3.4. For (1) we use the fact that the isomorphism $\psi=\psi_{(\beta, r, s)}: H_{n, t, \lambda} \rightarrow H_{n, t^{\prime}, \lambda^{\prime}}$ associated to the triple $(\beta, r, s) \in k^{*} \times \mathcal{U}\left(C_{n}, C_{2}\right) \times\{s \in\{1, \ldots, n-$ $1\} \mid \operatorname{gcd}(s, n)=1\}$ stabilizes $H_{4}$ if and only if $\beta=1$ and co-stabilizes $k\left[C_{n}\right]$ if and only if $s=1$.

Theorem 3.4 allows us to give a description of the automorphisms of $H_{4 n, \lambda, t}$. We denote, in what follows, by $v_{s}$ the automorphism of $C_{n}$ associated to $s \in\{1, \ldots, n-1\}, \operatorname{gcd}(s, n)=1$, given by $v_{s}\left(c^{i}\right)=c^{i s}$, for all $i$. We also note that $\left(\mathcal{U}\left(C_{n}, C_{2}\right), \cdot\right)$ is an Abelian group with point-wise multiplication. 
Corollary 3.7. The group $\operatorname{Aut}_{\mathrm{Hopf}}\left(H_{4 n, \lambda, t}\right)$ of all Hopf algebra automorphisms is parameterized by the set of all triples $(\beta, r, s) \in k^{*} \times \mathcal{U}\left(C_{n}, C_{2}\right) \times\{s \in\{1, \ldots, n-1\} \mid \operatorname{gcd}(s, n)=1\}$ satisfying (17) and (18) with $\left(\lambda^{\prime}, t^{\prime}\right)=(\lambda, t)$. The automorphism of $H_{4 n, \lambda, t}$ corresponding to $(\beta, r, s)$ is given for any $a \in H_{4}$ and $i=1, \ldots, n$ by

$$
\psi_{(\beta, r, s)}: H_{4 n, \lambda, t} \rightarrow H_{4 n, \lambda, t}, \quad \psi_{(\beta, r, s)}\left(a \# c^{i}\right)=u_{\beta}(a) r\left(c^{i}\right) \# v_{s}\left(c^{i}\right) .
$$

In particular, there exists an embedding

$$
\operatorname{Aut}_{\text {Hopf }}\left(H_{4 n, \lambda, t}\right) \hookrightarrow k^{*} \times\left(\mathcal{U}\left(C_{n}, C_{2}\right) \rtimes_{f} \operatorname{Aut}\left(C_{n}\right)\right),
$$

where $\mathcal{U}\left(C_{n}, C_{2}\right) \rtimes_{f} \operatorname{Aut}\left(C_{n}\right)$ is the semidirect product associated to $f: \operatorname{Aut}\left(C_{n}\right) \rightarrow \operatorname{Aut}\left(\mathcal{U}\left(C_{n}\right.\right.$, $\left.\left.C_{2}\right)\right), f(v)(p)=p \circ v$, for all $p \in \mathcal{U}\left(C_{n}, C_{2}\right)$ and $v \in \operatorname{Aut}\left(C_{n}\right)$.

Proof. The first assertion follows from Theorem 3.4. If $\psi_{(\alpha, p, q)}$ and $\psi_{(\beta, r, s)}$ are two automorphisms of $H_{4 n, \lambda, t}$, then, using the fact that $u_{\alpha}$ is a homomorphism which acts as the identity on $\{1, g\}$, we have

$$
\begin{aligned}
\psi_{(\alpha, p, q)} \circ \psi_{(\beta, r, s)}\left(a \# c^{i}\right) & =\psi_{(\alpha, p, q)}\left(u_{\beta}(a) r\left(c^{i}\right) \# v_{s}\left(c^{i}\right)\right)=u_{\alpha}\left(u_{\beta}(a) r\left(c^{i}\right)\right) p\left(c^{i s}\right) \# v_{q} v_{s}\left(c^{i}\right) \\
& =u_{\alpha \beta}(a) r\left(c^{i}\right)\left(p \circ v_{s}\right)\left(c^{i}\right) \# v_{q s}\left(c^{i}\right)=\psi_{\left(\alpha \beta, r \cdot\left(p \circ v_{s}\right), q s\right)}\left(a \# c^{i}\right) .
\end{aligned}
$$

This shows that $\Gamma: \operatorname{Aut}_{\text {Hopf }}\left(H_{4 n, \lambda, t}\right) \rightarrow k^{*} \times\left(\mathcal{U}\left(C_{n}, C_{2}\right) \rtimes_{f} \operatorname{Aut}\left(\mathbb{Z}_{n}\right)\right)$, defined by $\Gamma\left(\psi_{(\beta, r, s)}\right):=$ $\left(\beta,\left(r, v_{s}\right)^{-1}\right)$ is a one-to-one homomorphism of groups. Indeed,

$$
\begin{aligned}
\Gamma\left(\psi_{(\alpha, p, q)} \circ \psi_{(\beta, r, s)}\right) & =\Gamma\left(\psi_{\left(\alpha \beta, r \cdot\left(p \circ v_{s}\right), q s\right)}\right)=\left(\alpha \beta,\left(r \cdot\left(p \circ v_{s}\right), v_{q s}\right)^{-1}\right) \\
& =\left(\alpha \beta,\left(\left(r, v_{s}\right)\left(p, v_{q}\right)\right)^{-1}\right)=\left(\alpha,\left(p, v_{q}\right)^{-1}\right)\left(\beta,\left(r, v_{s}\right)^{-1}\right) \\
& =\Gamma\left(\psi_{(\alpha, p, q)}\right) \Gamma\left(\psi_{(\beta, r, s)}\right),
\end{aligned}
$$

which proves our claim and concludes the proof.

Example 3.8. As it can be easily seen from Theorem 3.6, the description of the classifying objects $\mathcal{H}^{2}\left(k\left[C_{n}\right], H_{4}\right)$ and $\operatorname{CRP}\left(k\left[C_{n}\right], H_{4}\right)$ depends on the arithmetics of the positive integer $n$. We describe below the two classifying objects for $n=2$ - this can serve as a model for other values of $n$. Let $k$ be a field of characteristic $\neq 2$. Then:

1. If $-1 \notin k^{2}$, then $\mathcal{H}^{2}\left(k\left[C_{2}\right], H_{4}\right) \cong \operatorname{CRP}\left(k\left[C_{2}\right], H_{4}\right)=\left\{H_{4} \otimes k\left[C_{2}\right]\right\}$.

2. If $-1=\zeta^{2}, \zeta \in k$, then $\mathcal{H}^{2}\left(k\left[C_{2}\right], H_{4}\right) \cong \operatorname{CRP}\left(k\left[C_{2}\right], H_{4}\right)=\left\{H_{4} \otimes k\left[C_{2}\right], H_{8, \zeta}\right\}$, where $H_{8, \zeta}$ is the 8-dimensional Hopf algebra having $\{1, g, x, g x, d, g d, x d, g x d\}$ as a $k$-basis, with the multiplication subject to the following relations

$$
g^{2}=1, \quad x^{2}=0, \quad d^{2}=g, \quad x g=-g x, \quad d g=g d, \quad d x=\zeta x d,
$$

and the coalgebra structure such that $g$ and $d$ are group-like elements and $x$ is $(1, g)$ primitive.

Indeed, $\mathcal{U}\left(C_{2}, C_{2}\right)=\left\{t_{1}, t_{2}\right\}$, where $t_{1}(c)=1$ and $t_{2}(c)=c$. Then $\sigma_{t_{1}}(2)=2$ and $\sigma_{t_{2}}(2)=3$. Thus,

$$
\mathcal{C S}(2, k)= \begin{cases}\left\{\left(t_{1}, 1\right),\left(t_{1},-1\right)\right\} & \text { if }-1 \notin k^{2}, \\ \left\{\left(t_{1}, 1\right),\left(t_{1},-1\right),\left(t_{2}, \zeta\right),\left(t_{2},-\zeta\right)\right\} & \text { if }-1=\zeta^{2}, \quad \zeta \in k .\end{cases}
$$

It is easy to see that $\left(t_{1}, 1\right) \approx\left(t_{1},-1\right)$ and $\left(t_{2}, \zeta\right) \approx\left(t_{2},-\zeta\right)$, the map $r \in \mathcal{U}\left(C_{2}, C_{2}\right)$ satisfying $(21)$ and (22), being, in both cases, $r=t_{2}$. Since the normalized 2-cocycles, $f_{t_{1}}$ and $f_{t_{2}}$, associated to $t_{1}$ and $t_{2}$ are not cohomologous, and the $s$ from (17) can take only the value 1 , we obtain using Remark 3.5, that $\left(t_{1}, 1\right) \not \equiv\left(t_{2}, \zeta\right)$. Thus, $\left(t_{1}, 1\right) \not \approx\left(t_{2}, \zeta\right)$, also. Finally, the description of $H_{8, \zeta}$ follows from Corollary 3.3. 


\section{Acknowledgements}

The authors would like to thank the referees for their comments and suggestions that substantially improved the first version of this paper. A.L. Agore is research fellow 'Aspirant' of FWO-Vlaanderen. This work was supported by a grant of the Romanian National Authority for Scientific Research, CNCS-UEFISCDI, grant no. 88/05.10.2011.

\section{References}

[1] Adem A., Milgram R.J., Cohomology of finite groups, Grundlehren der Mathematischen Wissenschaften, Vol. 309, 2nd ed., Springer-Verlag, Berlin, 2004.

[2] Agore A.L., Bontea C.G., Militaru G., Classifying coalgebra split extensions of Hopf algebras, J. Algebra Appl. 12 (2013), 1250227, 24 pages, arXiv:1207.0411.

[3] Agore A.L., Bontea C.G., Militaru G., Classifying bicrossed products of Hopf algebras, Algebr. Represent. Theory 17 (2014), 227-264, arXiv:1205.6110.

[4] Agore A.L., Militaru G., Extending structures II: The quantum version, J. Algebra 336 (2011), 321-341, arXiv:1011.2174.

[5] Andruskiewitsch N., Notes on extensions of Hopf algebras, Canad. J. Math. 48 (1996), 3-42.

[6] Andruskiewitsch N., Devoto J., Extensions of Hopf algebras, St. Petersburg Math. J. 7 (1996), $17-52$.

[7] Andruskiewitsch N., Natale S., Examples of self-dual Hopf algebras, J. Math. Sci. Univ. Tokyo 6 (1999), $181-215$.

[8] Andruskiewitsch N., Schneider H.J., Lifting of quantum linear spaces and pointed Hopf algebras of order $p^{3}$, J. Algebra 209 (1998), 658-691, math.QA/9803058.

[9] Brzeziński T., Hajac P.M., Coalgebra extensions and algebra coextensions of Galois type, Comm. Algebra 27 (1999), 1347-1367, q-alg/9708010.

[10] García G.A., Vay C., Hopf algebras of dimension 16, Algebr. Represent. Theory 13 (2010), 383-405, arXiv:0712.0405.

[11] Krop L., Classification of isomorphism types of Hopf algebras in a class of Abelian extensions, arXiv:1211.5621.

[12] Masuoka A., Hopf algebra extensions and cohomology, in New Directions in Hopf Algebras, Math. Sci. Res. Inst. Publ., Vol. 43, Cambridge University Press, Cambridge, 2002, 167-209.

[13] Montgomery S., Hopf algebras and their actions on rings, CBMS Regional Conference Series in Mathematics, Vol. 82, Amer. Math. Soc., Providence, RI, 1993.

[14] Sweedler M.E., Cohomology of algebras over Hopf algebras, Trans. Amer. Math. Soc. 133 (1968), 205-239. 